\title{
Crystal-Storing Histiocytosis with Plasma Cell Neoplasm in the Setting of Chronic Carbamazepine Exposure
}

\author{
Woo Cheal Cho · Safina Hafeez · Peter Shen \\ Department of Pathology and Laboratory Medicine, Hartford Hospital, Hartford, CT, USA
}

Crystal-storing histiocytosis (CSH) is an uncommon phenomenon in which crystalline material accumulates within the cytoplasm of histiocytes, and is typically associated with an underlying lymphoproliferative or plasma cell disorder with monoclonal immunoglobulin expression, such as multiple myeloma (MM), lymphoplasmacytic lymphoma (LPL), and monoclonal gammopathy of undetermined significance (MGUS). ${ }^{1,2}$ With a few exceptions, the crystalline materials are almost exclusively composed of $\kappa$ immunoglobulin light chains. ${ }^{2,3}$ Recently, other less common variants of $\mathrm{CSH}$ in which the crystalline material is not of an immunoglobulin origin have also been described, including clofazimine-induced $\mathrm{CSH}^{4,5}$ Here, we describe a case of $\mathrm{CSH}$ with plasma cell myeloma in the setting of chronic carbamazepine exposure.

\section{CASE REPORT}

A 72-year-old woman with a history of chronic renal insufficiency, hypothyroidism, peripheral neuropathy, hypercholesterolemia, anxiety, and tremor was referred from her primary care physician's office to the emergency department for evaluation of pancytopenia. Her peripheral neuropathy had been managed with carbamazepine for several years. Bone marrow biopsy revealed infiltration and near-total replacement by cells filled with eosinophilic crystalloid material (Fig. 1A, B). Hematopoietic cells

Corresponding Author

Woo Cheal Cho, MD

Department of Pathology and Laboratory Medicine, Hartford Hospital, 80 Seymour Street, Hartford, CT 06102-5037, USA

Tel: +1-860-972-2488, Fax: +1-860-545-2204

E-mail:WooCheal.Cho@hhchealth.org

Received: April 10, 2018 Revised: May 24, 2018

Accepted: May 25, 2018 were virtually undetectable, and extensive reticulin fibrosis was noted. CD138-positive, $\kappa$-restricted plasma cells (Fig. 1C) were highlighted to form collections and aggregates in the marrow, comprising at least $30 \%$ of total cellularity. No definitive $\operatorname{IgG}$, $\operatorname{IgA}$, or IgM was observed within the plasma cells. Histiocytes comprised the remainder of the cell population and were highlighted by CD68 (Fig. 1D) and CD163. $\kappa$ expression (Fig. 1E) was also noted within the histiocytes. CD34 and CD117 failed to reveal a significant blast population. Other than occasional expressions, no significant myeloid population was highlighted by CD15. No CD1a expression was observed within histiocytes/ macrophages. No metastatic carcinoma was revealed by cytokeratin. CD61 also failed to reveal the presence of megakaryocytes.

By polychromatic flow cytometry, approximately $58 \%$ of cells in the specimen displayed the phenotypic and light scatter properties of lymphocytes. Most of these were T cells, without specific phenotypic abnormalities, including CD4-positive and CD8-positive subsets (CD4:CD8 ratio of 2.5:1). B cells comprised approximately $12 \%$ of cells. No normal B-cell precursors (hematogones) were seen. Phenotypically mature B cells comprised a mixture of $\kappa$ - and $\lambda$-bearing cells, without evidence of light chain restriction. Cells with the phenotypic and light scatter properties characteristic of plasma cells comprised $4 \%$ of cells, and included predominantly a cytoplasmic $\kappa$-restricted plasma cell population. No definitive expression for $\operatorname{IgA}$, IgG, IgD, and IgM was noted within the plasma cells. CD34-positive myeloblasts comprised approximately $<1 \%$ of cells. Very few granulocytic cells were observed (17\% of total cells), and $5 \%$ nucleated red blood cells were seen.

Further workup including serum protein and immuno-fixation electrophoresis showed hypogammaglobulinemia and markedly elevated monoclonal free $\kappa$ light chains with a free $\kappa \cdot \lambda$ ratio of 


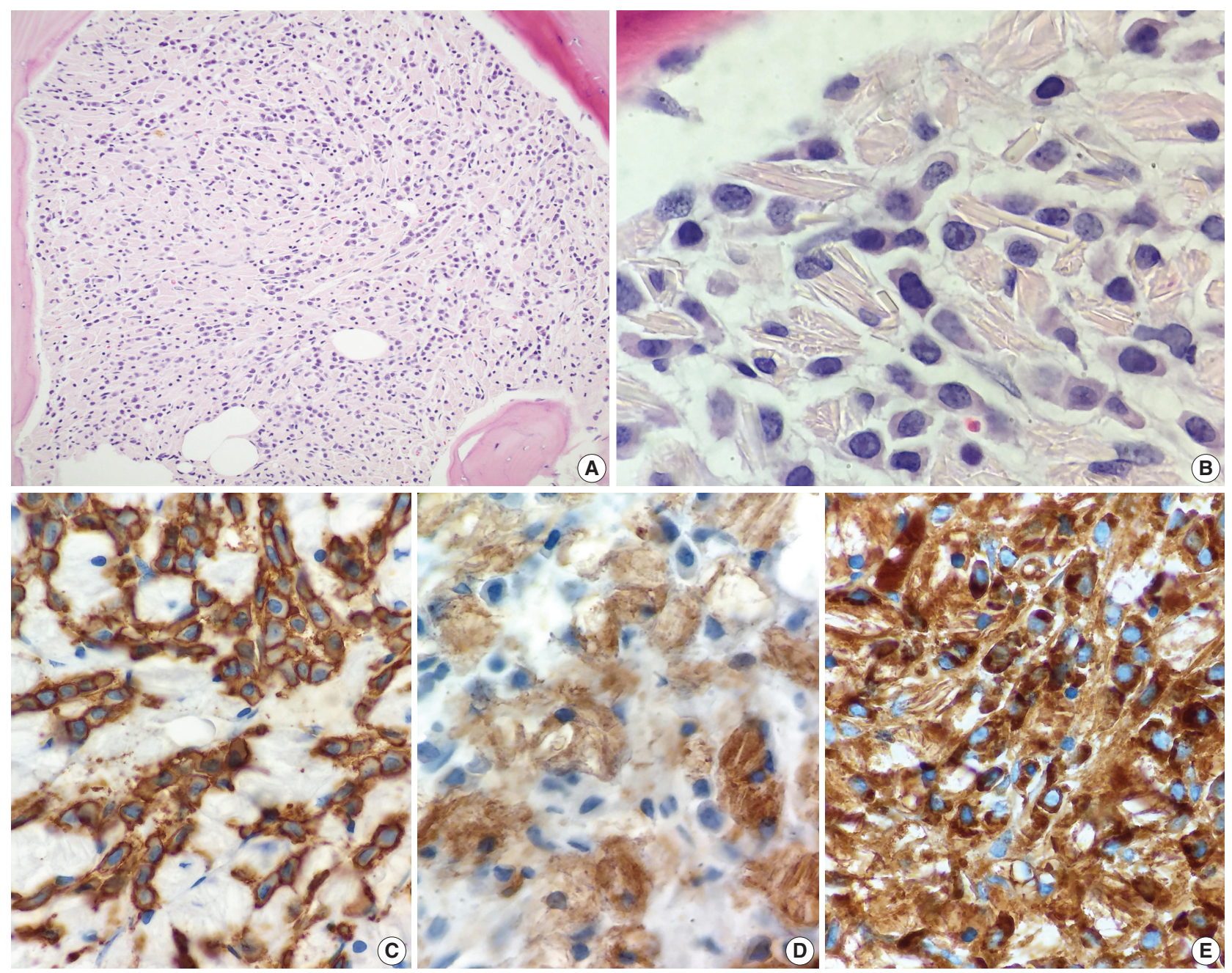

Fig. 1. Histologic findings of bone marrow biopsy. (A, B) Bone marrow biopsy showed infiltration and near-total replacement by cells filled with eosinophilic crystalloid material. The neoplastic cells were CD138-positive (C), kappa-restricted plasma cells, while the remainder of the cell population were histiocytes highlighted by CD68 (D) and CD163 (not shown). (E) Kappa expression was also noted within the histiocytes.

more than 700:1. These findings were most consistent with CSH associated with plasma cell myeloma. The patient was immediately placed on bortezomib and dexamethasone. Subsequent skeletal survey revealed no significant evidence of lytic lesions.

Formal written informed consent was not required with a waiver by the appropriate Institutional Review Board of Hartford Hospital (FWA00021932).

\section{DISCUSSION}

$\mathrm{CSH}$ is a rare, but well-documented phenomenon most frequently seen in patients with an underlying lymphoproliferative or plasma cell disorder with monoclonal immunoglobulin expression, such as MM, LPL, and MGUS ${ }^{1,2}$ Other less common variants of $\mathrm{CSH}$ that are not of an immunoglobulin origin, including clofazimine-induced CSH, have also recently been described. ${ }^{4,5}$ Clofazimine is a drug used to treat leprosy with frequent side effects including red to brownish discoloration of the skin and gastrointestinal disturbances. Reportedly, prolonged treatment with higher-dose clofazimine can also lead to $\mathrm{CSH}$, albeit rare, in which clofazimine crystals accumulate in the lamina propria of the small bowel and may result in a severe enteropathy. ${ }^{4}$ Clofazimine crystals appear clear in routine histologic sections, as they dissolve in alcohol and other organic solvents, and in the presence of background plasmacytosis, may simulate histologically other immunoglobulin-associated CSHs. ${ }^{4}$ Our patient in the presented case had been taking carbamazepine, an antiepileptic drug, for several years for management of peripheral neuropathy. Currently, there is no known evidence of a direct link between prolonged carbamazepine exposure and development of CSH. However, 
immune abnormalities are well-documented long-term adverse effects of antiepileptic drugs; and, in fact, development of hematologic disorders, including non-Hodgkin's lymphoma and MM, following a prolonged treatment with antiepileptic therapy, particularly phenytoin, has previously been reported. ${ }^{6}$ The potential carcinogenic effect of carbamazepine is still unclear, although pancytopenia as in our patient has been rarely observed in patients receiving carbamazepine. Carbamazepine can alter the immune system by increasing the production of various interleukins (ILs), including IL-6, which is known to play a key role in the pathogenesis of MM. IL-6 not only serves as a growth factor, but it also serves as a survival factor in MM, ultimately inhibiting apoptosis of myeloma cells. ${ }^{7,8}$ Recently, there have been a few case reports raising the possibility of a causal relationship between carbamazepine exposure and subsequent development of hypogammaglobulinemia, MGUS or MM. ${ }^{9,10}$ These reports are still anecdotal and few in number, and further studies are needed to fully understand the potential carcinogenicity of carbamazepine and its possible link with plasma cell neoplasms.

In conclusion, we report a unique case of $\mathrm{CSH}$ with plasma cell myeloma thought to have developed following prolonged carbamazepine therapy. Although plasma cell neoplasms have been reported in patients with prolonged exposure to carbamazepine, to the best of our knowledge, no case of $\mathrm{CSH}$ with plasma cell neoplasm in the setting of chronic carbamazepine exposure has been previously described.

\section{ORCID}

Woo Cheal Cho: https://orcid.org/0000-0001-5867-1403

\section{Author Contributions}

Conceptualization: WCC.

Data curation: WCC, PS.

Formal analysis: WCC, SH, PS.

Investigation: WCC, SH.

Methodology: PS.

Project administration: WCC, PS.

Resources: SH.

Supervision: PS.

Validation: WCC.
Writing—original draft: WCC.

Writing-review \& editing: WCC, PS.

\section{Conflicts of Interest}

The authors declare that they have no potential conflicts of interest.

\section{REFERENCES}

1. Kapadia SB, Enzinger FM, Heffner DK, Hyams VJ, Frizzera G. Crystal-storing histiocytosis associated with lymphoplasmacytic neoplasms: report of three cases mimicking adult rhabdomyoma. Am J Surg Pathol 1993; 17: 461-7.

2. Lebeau A, Zeindl-Eberhart E, Müller EC, et al. Generalized crystalstoring histiocytosis associated with monoclonal gammopathy: molecular analysis of a disorder with rapid clinical course and review of the literature. Blood 2002; 100: 1817-27.

3. Jones D, Bhatia VK, Krausz T, Pinkus GS. Crystal-storing histiocytosis: a disorder occurring in plasmacytic tumors expressing immunoglobulin kappa light chain. Hum Pathol 1999; 30: 1441-8.

4. Sukpanichnant S, Hargrove NS, Kachintorn U, et al. Clofazimineinduced crystal-storing histiocytosis producing chronic abdominal pain in a leprosy patient. Am J Surg Pathol 2000; 24: 129-35.

5. Dogan S, Barnes L, Cruz-Vetrano WP. Crystal-storing histiocytosis: report of a case, review of the literature ( 80 cases) and a proposed classification. Head Neck Pathol 2012; 6: 111-20.

6. Olsen JH, Schulgen G, Boice JD Jr, et al. Antiepileptic treatment and risk for hepatobiliary cancer and malignant lymphoma. Cancer Res 1995; 55: 294-7.

7. Gado K, Domjan G, Hegyesi H, Falus A. Role of INTERLEUKIN-6 in the pathogenesis of multiple myeloma. Cell Biol Int 2000; 24: 195-209.

8. Verrotti A, Basciani F, Trotta D, Greco R, Morgese G, Chiarelli F. Effect of anticonvulsant drugs on interleukins- $1,-2$ and -6 and monocyte chemoattractant protein-1. Clin Exp Med 2001; 1: 133-6.

9. Moreno-Ancillo A, Cosmes Martin PM, Dominguez-Noche C, et al. Carbamazepine induced transient monoclonal gammopathy and immunodeficiency. Allergol Immunopathol (Madr) 2004; 32: 86-8.

10. Günaldı M, Paydaş S, Usul Afşar C, Bozkurt Duman B, Erçolak V, Haksöyler V. Carbamazepine and multiple myeloma: possible interaction. Turk J Haematol 2013; 30: 83-4. 